Article

\title{
Experimental Fitting of the Re-Scaled Balje Maps for Low-Reynolds Radial Turbomachinery
}

\author{
Roberto Capata * and Enrico Sciubba \\ Department of Mechanical and Aerospace Engineering, University of Roma "Sapienza", \\ Roma 00184, Italy; E-Mail: enrico.sciubba@uniroma1.it \\ * Author to whom correspondence should be addressed; E-Mail: roberto.capata@uniroma1.it; \\ Tel.: +39-064-458-5243; Fax: +39-064-458-5850.
}

Academic Editor: K. T. Chau

Received: 9 February 2015 / Accepted: 22 July 2015 / Published: 31 July 2015

\begin{abstract}
With the increasing popularity enjoyed by ultra-micro scale turbomachinery, designers are often faced with severe challenges due to the substantial phenomenological difference between the low-Reynolds fluid-dynamics in rotating or strongly curved flows and the established knowledge acquired through decades of theoretical and experimental studies on medium and large-scale machines. The problem is complicated by the absence of an extended and reliable database that might be used for preliminary design and provide indications for scale-up or scale-down. As a result, custom-designed experimental campaigns are necessary that make the development of any new machine exceedingly costly. The situation has seen some improvement in recent years, after the publication of a sufficient number of experimental results and numerical simulations that pave the way towards a development of semi-empirical correlations. The purpose of this work is to present and discuss a preliminary and simple method to extend the currently available design maps into the small scale range $\left(\operatorname{Re}<10^{5}\right)$ by introducing in the Balje charts an efficiency correction that depends on the specific speed $n_{\mathrm{s}}$. The method results in a Stodola-like formula which originates a lower-than-standard Cordier curve on the classical Balje charts. A validation with some experimental results is also presented and discussed. Though the agreement is more than satisfactory, it must be stressed that the method provides only approximate results, and thus it must be considered as an evolving temporary solution, that needs to be updated as long as larger series of (numerical or physical) experimental results become available.
\end{abstract}


Keywords: Balje maps; Stodola efficiency formula; ultra-micro gas turbine; turbomachinery design

\section{Introduction}

In the early design stages, an (approximately) correct prediction of the performance of a turbomachine is critical, not only because it ensures that the type of components of the stage (impeller, diffuser and volute) are in line with current technology and with the design specifications, but also because it facilitates the subsequent final design task. The general performance maps available from manufacturers are usually limited to a small range of rotational speeds, pressure ratios and mass flow rates, and extrapolating on their basis is especially dangerous at the onset of a selection or design task. A comprehensive prediction procedure would enable the designer to analyze the performance of all the individual components already at the initial design stage, and would obviously result in an "optimal" design with a lower effort in terms of human and computational resources. Advanced computational fluid dynamics (CFD) procedures are better suited for later design stages, and one-dimensional prediction techniques continue to be the most practical method of predicting the performance of a stage or of one of its components [1]. Since the early works by Wiesner [2] and Stodola and Lowestein [3], one-dimensional prediction techniques and related semi-empirical loss correlations have been persistently developed [4-7]. The prevailing approach is to lump together losses of different type in the form of either a slip factor, a loss coefficient, or both. The downside of such a model is that the effects of individual losses are not clearly characterized and some phenomenological information is lost. This paper deals solely with polytropic losses in each component of a compressor (thermal phenomena linked to the non-adiabaticity of the flow being neglected) and lumps them together to calculate in a simple and convenient fashion their total extent. The model discussed here is based on the old idea of separating the losses in an "inviscid" and a "viscous" part, and subtracting them from the inviscid Euler work. In this perspective, the Reynolds number influence on the performance of turbomachinery has been the object of considerable attention in the technical literature, and several more or less general - and more or less reliable-models have been developed for both incompressible and compressible flow machines. Several proposals of more or less general models of an efficiency scaling of the type $\eta=f(R e)$ have been published, but all of the proposed models agree with the experimental data only within a limited range of configurations and fall therefore short of representing general design correlations. An acknowledged problem of the available formulations is the complexity and the non-crispness of the assumed phenomenological model. All approaches-except for the very detailed and costly 3-D, fully turbulent numerical simulations-lack sufficient accuracy and generality in separating Reynolds dependent and independent losses, and this makes it very difficult to quantify the Re-independent loss fraction, that clearly depends on manufacturing methods, tolerances and clearances. Most studies in this field are based on the classical but dated experiments carried out by Wiesner [2], Casey [8], Strub et al. [9] and others. The actual modelling varies somewhat from author to author, but a common starting point is the definition of a reference Reynolds number based on the rotor exit passage width: 


$$
\operatorname{Re}_{b 2}=\frac{U_{2} b_{2}}{v}
$$

Since the aim of this paper is to search for a possible correction factor to the Balje charts, our definition of the reference Re is at variance with Equation (1), and is based on the rotor outer diameter, like in Balje's approach [4]:

$$
\operatorname{Re}_{D 2}=\frac{U_{2} D_{2}}{v}
$$

Some scaling formulations [2,6,8,9] include the influence of Reynolds number and surface roughness, but in this work only hydraulically smooth surfaces are considered, to eliminate roughness from the set of relevant variables: this choice was in fact almost unavoidable, given the scarcity of data available on the prototypal rotors in the ultra-micro scale range. We are very well aware of the influence of surface finishing in real machines, but suggest leaving the evaluation of its effects to specific lab-scale tests, easily performed on small-scale machines: we anticipate the possibility of reporting in a future work a possibly parametric correlation between efficiency and surface roughness. In the same line of reasoning, the effects of the non-adiabaticity [1] are neglected. In spite of these quite radical simplifications, the derivation of a "universal" formula is intrinsically difficult, due to the substantial difference in the flow phenomenology, so that each class of machines requires a specific analysis. The scaling formula suggested here has indeed (as most of the Re-correction formulae found in literature) the same structure as Wiesner's formula, the novelty residing in the fact that its coefficients have been derived via numerical simulations and validated by a series of laboratory tests. Such a double-calibration, so to say, makes it a valuable extension of the existing design guidelines. The formula is not intended to cover the entire range of "low-Re machines": in fact, its verified range of validity falls between $\operatorname{Re}_{D 2}=1 \times 10^{4}$ and $1 \times 10^{5}$, which is common for ultra-micro machines $\left(\mathrm{Re} \cong 10^{5}\right)$. In the following, the suffix " 2 " in " $D_{2}$ " is omitted.

\section{Reynolds Number Effects}

The general paradigm for all Re-correction formulae is the so-called Stodola [8] correlation:

$$
\frac{1-\eta}{1-\eta_{\text {ref }}}=\mathrm{a}+(1-\mathrm{a}) \cdot\left[\frac{\mathrm{Re}_{\text {ref }}}{\mathrm{Re}}\right]^{n}
$$

The coefficients $a$ and $n$ differ from author to author, as shown in Table 1, and represent the Re-independent loss fraction, which is are mainly related to curvature and rotation effects (relative vortex). Leakage losses, that are not really viscosity-independent, are usually accounted for by assuming that $a$ is actually constant only in a small range of Re but that it may vary to a lesser extent with both geometry and manufacturing techniques even for machines with the same Re. It turns out, that $a$ has in fact sometimes been found to be itself a function of Re, while there is a general agreement on the exponent $n$ being inversely proportional to the Reynolds number. The usual practice, that we shall also follow, is to assume that the manufacturing process and clearances are in line with those specified by the Balje charts, so that a remains constant for the range denoted here as the "micro-machine scales", i.e., $1 \times 10^{4}<\operatorname{Re}_{D}<1 \times 10^{5}$. As for the exponent $n$, its value varies considerably from author to author, and though this in part may depend on the different intrinsic accuracy of the data [10], a more plausible 
explanation is that it, too, displays a Re-dependence: Wiesner [2] was the first author to postulate an explicit Re-dependence, and proposed a functional relation of the form:

$$
n=\mathrm{c}^{\prime}\left(\frac{1}{\operatorname{Re} / \operatorname{Re}_{\text {ref }}}\right)^{\mathrm{c}}
$$

Remarkably, very few authors provide in their papers precise indications about the Re validity range (thence the "n.d." in Table 1): a careful inspection of the available graphs indicates that most formulae are applicable between $1 \times 10^{4}$ and $1 \times 10^{5}$, with Wiesner being the only author to supply a well-defined range: $5 \times 10^{4} \div 5 \times 10^{5}$. As already reported by Balje, the pressure difference between suction and pressure sides causes a non-negligible amount of leakage over the blade tip.

Table 1. Summary of the most popular efficiency correction equations [2].

\begin{tabular}{|c|c|c|c|c|c|c|}
\hline Year & Source & $\begin{array}{l}\text { Inviscid loss } \\
\text { fraction "a" }\end{array}$ & $\begin{array}{c}\text { Viscosity-dependent loss } \\
\text { fraction }(1-a) \\
\end{array}$ & Exponent $n$ & Re range & Machine type \\
\hline 1925 & Moody & 0.25 & 0.75 & 0.33 & n.d. & Propeller turbines \\
\hline 1930 & Ackeret \& Muhlemann & 0.50 & 0.50 & 0.20 & n.d. & Hydraulic turbines \\
\hline 1942 & Moody & 0.00 & 1.00 & 0.20 & n.d. & Pumps \\
\hline 1947 & Pfleiderer & 0.00 & 1.00 & 0.10 & n.d. & Pumps \\
\hline 1951 & Davis, Kottas \& Moody & 0.00 & 1.00 & Variable & n.d. & All turbomachines \\
\hline 1954 & Hutton & 0.30 & 0.70 & 0.20 & n.d. & Kaplan turbines \\
\hline 1958 & Rotzoll & 0.00 & 1.00 & Variable & n.d. & Pumps \\
\hline \multirow{2}{*}{1960} & Wiesner & 0.50 & 0.50 & 0.10 & \multirow{2}{*}{$\begin{array}{c}5 \times 10^{4} \div \\
5 \times 10^{5}\end{array}$} & \multirow{2}{*}{ Radial compressors } \\
\hline & Fauconnet & 0.24 & 0.76 & 0.20 & & \\
\hline 1961 & O’Neil \& Wickli & 0.00 & 1.00 & Variable & n.d. & Radial compressors \\
\hline \multirow{2}{*}{1965} & \multirow{2}{*}{ ASME Code PTC-10 } & 0.00 & 1.00 & 0.20 & \multirow{2}{*}{ n.d. } & Axial compressors \\
\hline & & 0.00 & 1.00 & 0.10 & & Radial compressors \\
\hline 1971 & Mashimo et al. & $0.25 \mathrm{~min}$ & $0.75 \max$ & 0.20 & n.d. & Radial compressors \\
\hline 1974 & Mashimo et al. & $0.15-0.57$ & $0.43-0.85$ & $0.20-0.50$ & n.d. & Radial compressors \\
\hline
\end{tabular}

This effect depends on the blade geometry, the clearance gap and the load. For what leakage is concerned, the non-Re related losses depend on other parameters, which scale according to different similarity rules, such as turbulence level, roughness effects, etc. Balje himself suggests the adoption of a "correction factor" for adjusting the turbo machinery efficiency. This consideration was included in our calculations.

\section{Calculation of the Correlation Coefficients}

It is quite apparent that Equations (3) and (4) are "complex" enough (i.e., they subsume a sufficiently deep phenomenological model) to yield a reasonably accurate estimate of the losses for a particular class of machines such as the ultra-micro ones considered here. Due to the fact that ultra-micro turbomachinery has only been developed in relatively recent times, and in contrast to what happens for large-scale machines, a sufficiently large experimental database for coefficient fitting does not exist, which led us to the idea of adopting a "hybrid" fitting procedure, which consists in first deriving the coefficients via relatively inexpensive numerical simulations, and then verifying them by 
means of the available (limited) experimental database. To this purpose, a sizeable set of data was created by means of sufficiently accurate numerical simulations [11-14], to derive initial values for the constants a, c and c' in Equations (3) and (4). The simulations — discussed in detail elsewhere [11] —were performed on 3-D models in kinematic similarity using a commercial CFD simulation code, ANSYS/Fluent. The turbulence model was the $k$ - $\varepsilon$ realizable, with second order accuracy. Each model was meshed to ensure a $y^{+}{ }_{\max } \sim 5$, a necessary condition for adopting the enhanced wall treatment, since the quality of the grid has a relevant importance on the accuracy and stability of the numerical simulation. Commercial software allows the "plastering" of cell layers to the critical boundaries of the control volume, which are obviously, in this case, the wall surfaces of the hub, casing and blades. In these zones the usual practice is that of creating a completely structured boundary layer, specifying whenever possible both the height of the first row of cells and the "growth ratio", i.e. the rate that determines the height of the successive cells. In this process, the height of the first row of cells is usually determined via an empirical formula that gives the value of a wall-based local Reynolds number, denoted by $y^{+}$:

$$
y^{+}=u^{*} \cdot \frac{y}{v}
$$

where $u^{*}=\sqrt{\frac{\tau_{\text {wall }}}{\rho}}, \tau_{\text {wall }}$ being the wall shear stress. For both turbine and compressor the procedure was the same: the stator control volume was split in several smaller sub-volumes, to achieve a more consistent set of faces and to better exploit the possibility of creating a locally more refined grid. The choice of the boundary conditions was made more difficult by the lack of conclusive experimental data: it was performed heuristically, starting from the preliminary sizing data, calibrating them by means of a first simulation, adjusting the values by iteratively resetting the outlet static pressure on the near-wake radial area downstream of the trailing edge. Through subsequent simulations the values of the inlet total pressure and temperature were refined as well in order to ensure conservation of the mass flow rate (the so-called "mass flow inlet condition" was adopted). The turbulent parameters were the hydraulic diameter $D$ hyd and the turbulence intensity $\boldsymbol{I}=\sqrt{\boldsymbol{k}} / \boldsymbol{U}$. Rotational periodicity was imposed on all lateral channel surfaces. As discussed below, the resulting model retains its validity for compressors and turbines with diameter down to the order of millimetres, without the need for a re-calibration of the constants. The data extracted from the numerical simulations $[11,12,14]$ of the ultra micro-compressor and turbine were used to calibrate the formula coefficients a, c, c'. The main dimensional characteristic of both component are shown in the simplified geometry of Figure $1[11,13]$.

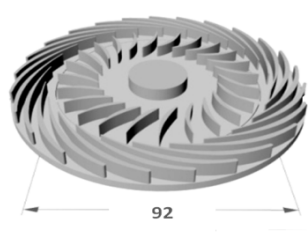

(a)

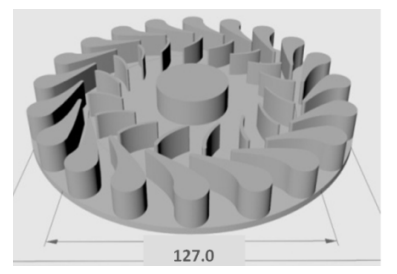

(b)

Figure 1. (a) 3-D sketch reference geometry for ultra micro gas turbine generator (UMGTG) compressor, where $D_{\mathrm{e}}=92.0 \mathrm{~mm}[11,13]$; (b) reference geometry for turbine, $D_{\mathrm{e}}=127.0 \mathrm{~mm}[11]$. 
To study the effect of the Reynolds number on the viscous-related losses, we simulated three models of the original device at different scales $(1: 3,1: 5$ and 1:10). The data are reported in Table 2. The largest model is taken as the reference geometry (it falls within the range of the "commercial scales"). Table 2 indicates that the considered compressors and turbine are outside of the optimal $n_{\mathrm{s}} / d_{\mathrm{s}}$ range. In fact, the Re of our 1:1 model falls within the range indicated by the Balje maps (Figure 2), where it displays a rather low efficiency, which was expected, because the adopted geometry is based on an "extruded" 2-D blade shape with zero twist.

Table 2. Data for different compressor and turbine models (all dimensions in $\mathrm{mm}$ ).

\begin{tabular}{cccccccc}
\hline \multicolumn{7}{c}{ Compressors } \\
\hline Scale & $\left.\boldsymbol{D}_{\mathbf{2}} \mathbf{( m m}\right)$ & $\boldsymbol{b}_{\mathbf{2}}(\mathbf{m m})$ & $\boldsymbol{\omega}(\mathbf{r a d} / \mathbf{s})$ & $\boldsymbol{n}_{\mathbf{s}}$ & $\boldsymbol{d}_{\mathbf{s}}$ & $\boldsymbol{\eta}_{\mathbf{s t}}(\mathbf{\%})$ & $\mathbf{R e}_{\mathbf{R} \mathbf{e}_{\text {ref }}}$ \\
\hline $1: 10$ & 9.2 & 0.55 & 68745 & 0.38 & 5.7 & 52.5 & $1 / 10$ \\
$1: 3$ & 27.6 & 1.65 & 65217 & 0.39 & 6.2 & 57.9 & $3 / 10$ \\
$1: 5$ & 46.0 & 2.75 & 63376 & 0.40 & 7.2 & 59.2 & $2 / 10$ \\
$1: 1$ & 92.0 & 5.50 & 57160 & 0.42 & 8.1 & 60.6 & 1 \\
\hline \multicolumn{7}{c}{ Turbine } \\
\hline Scale & $\boldsymbol{D}_{\mathbf{1}}(\mathbf{m m})$ & $\boldsymbol{b}_{\mathbf{1}}(\mathbf{m m})$ & $\boldsymbol{\omega}(\mathbf{r a d} / \mathbf{s})$ & $\boldsymbol{n}_{\mathbf{s}}$ & $\boldsymbol{d}_{\mathbf{s}}$ & $\boldsymbol{\eta}_{\mathbf{s t}}(\mathbf{\%})$ & $\mathbf{R e}_{\mathbf{R}} \mathbf{R e}_{\text {ref }}$ \\
\hline $1: 10$ & 12.7 & 1.31 & 68745 & 0.50 & 3.99 & 75.0 & $1 / 10$ \\
$1: 3$ & 38.1 & 3.93 & 65217 & 0.47 & 4.20 & 76.1 & $3 / 10$ \\
$1: 5$ & 63.5 & 6.55 & 63376 & 0.46 & 4.32 & 79.3 & $2 / 10$ \\
$1: 1$ & 127 & 13.10 & 57160 & 0.41 & 4.91 & 80.0 & 1 \\
\hline
\end{tabular}

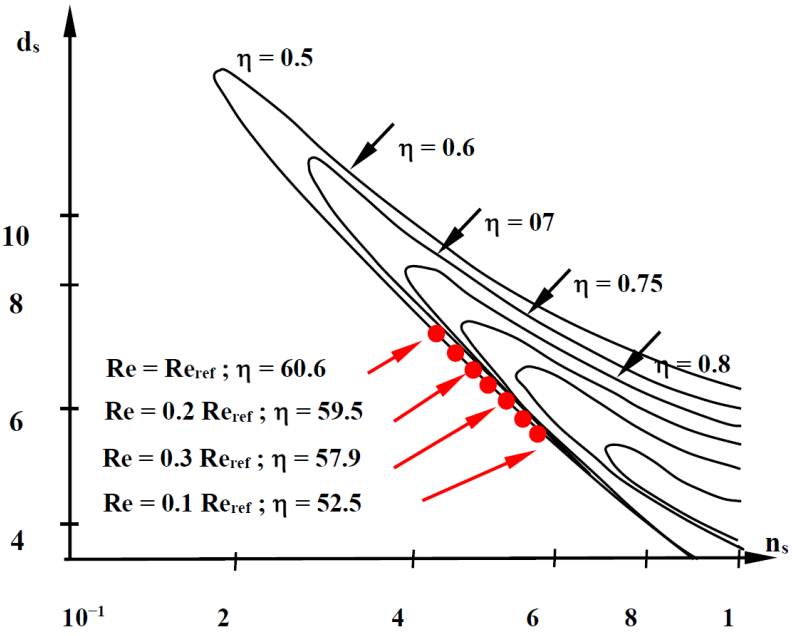

(a)

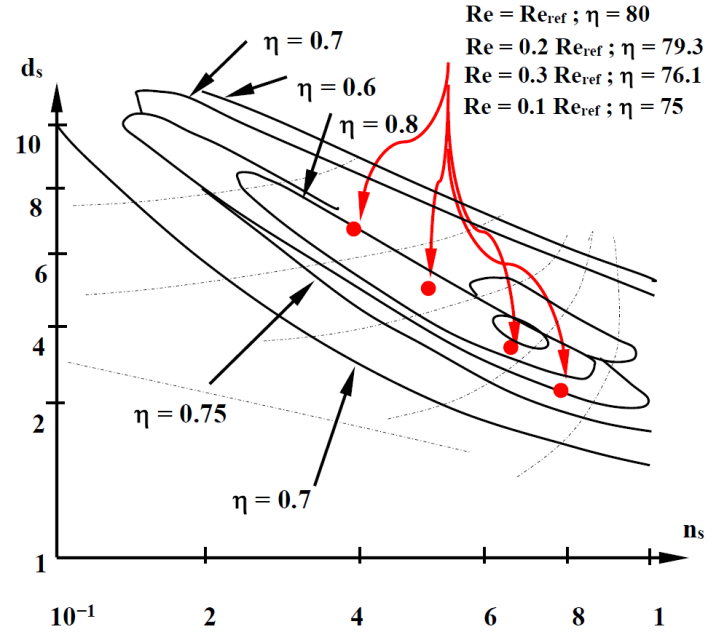

(b)

Figure 2. Computed efficiencies $n_{\mathrm{s}}$ for (a) turbine and (b) compressor . $\mathrm{Re}_{\mathrm{ref}}=10^{6}$.

By fitting the computed data we obtained a value 0.50 for $a$, and respectively 0.25 and 0.084 for $c$ and $c^{\prime}$. More precisely, the value of $a$ was calculated (to the third significant digit) using the method of the bisections for every single pair of data points. The input for the method are the simulation values, in an interval $[p, q]$, and the function values $\mathrm{f}(p)$ and $\mathrm{f}(q)$. The function values are of opposite sign (there is at least one zero within the interval). Each iteration performs these steps:

(1) Calculate $x$, the midpoint of the interval, $x=0.5 \cdot(p+q)$;

(2) Calculate the function value at the midpoint, $\mathrm{f}(x)$; 
(3) If convergence is satisfactory (that is, $p-x$ is sufficiently small, or $\mathrm{f}(x)$ is sufficiently small), return $x$ and stop iterating;

(4) Examine the sign of $\mathrm{f}(x)$ and replace either $(p, \mathrm{f}(p))$ or $(q, \mathrm{f}(q))$ with $(x, \mathrm{f}(x))$ so that there is a zero crossing within the new interval.

Thus the final formula we suggest and propose for the scale-down is:

$$
\frac{1-\eta}{1-\eta_{\text {ref }}}=0.50+0.50 \cdot\left[\frac{\operatorname{Re}_{\text {ref }}}{\operatorname{Re}}\right]\left[0.084 \cdot\left(\frac{\mathrm{Re}_{\mathrm{ref}}}{\mathrm{Re}}\right)^{0.25}\right]
$$

The coefficients in Equation (5) provide a good agreement with the values reported in literature, particularly with those of Wiesner. Figure 3 reports the location of the points obtained by applying Equation (5) to several ultra-micro gas turbine devices designed by several independent research groups $[10,12,15]$.

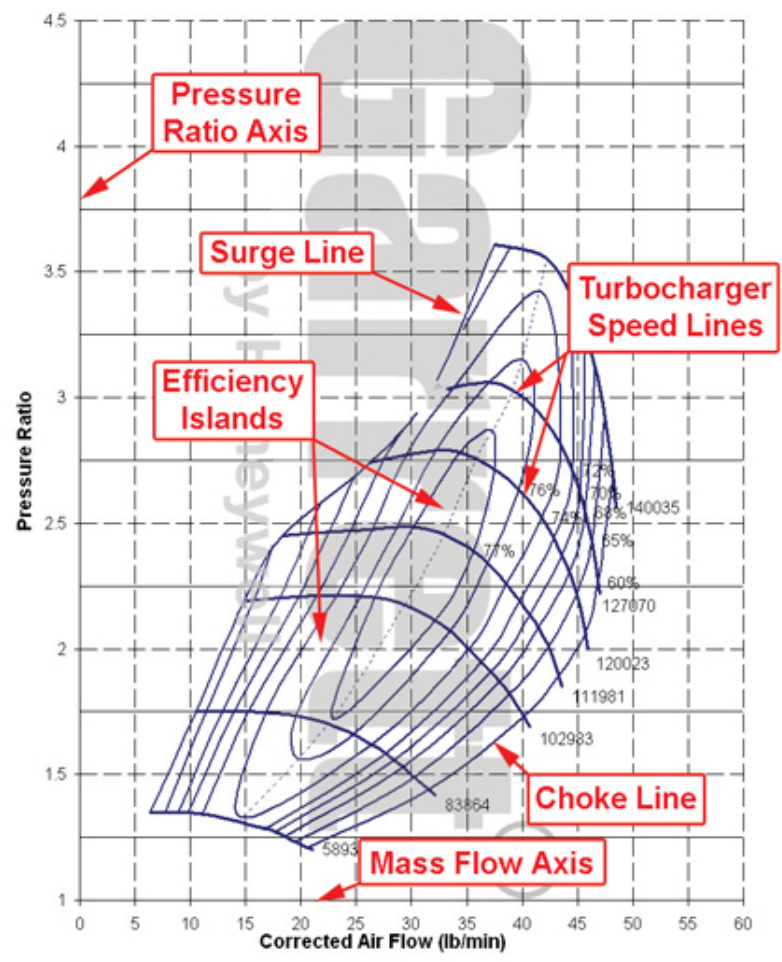

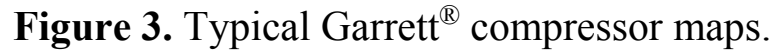

\section{Results of the Computational Fluid Dynamics Validation}

A series of CFD simulations was necessary to obtain the data needed to reconstruct the correlations presented here: more details are offered in previous articles by the same group $[11,13,14,16]$, so that only some essential considerations will be reported here. The analysis compared the collected data to the resulting efficiency predicted by the charts for the given $n_{\mathrm{s}}$ and $d_{\mathrm{s}}$. Both correlations predict an efficiency of the 1:10 model remarkably different from the one predicted by the Balje charts (60.6\% and $75 \%$ versus $78 \%$ and $88 \%$ for compressor and turbine respectively). Since the charts refer to well-designed, commercial scale configurations, this difference is likely to be related to both the machine geometry and size. On the basis of the data reported by Wiesner [2], in which different 
small-sized compressors with 2-D and 3-D geometries were compared, it can be concluded that - all other parameters being the same - the penalty brought about by a "2-D" stage with respect to a 3-D one is about $18 \%$. This constitutes an indirect validation for the correlations derived by our numerical calculations. In fact, $0.75 \times 0.82=0.615$, which is very close to our 0.606 , where 0.82 is the efficiency deduced by the Balje chart and 0.606 is the efficiency calculated by the simulations. It seems to indicate that the penalty due to a non-optimal geometry can be modeled by a function of the Reynolds number in which at least one of the coefficients is geometry dependent.

\section{Validation of the Correlation on Commercial Ultra-Micro Machines}

The goal of our study was to generate a procedure for preliminary design applications. What is proposed here is to use Equation (3) to scale the efficiency with the Reynolds number calculated as in Equation (2). The coefficients in Equation (3) were been calculated, as described above, by an accurate fitting of the results of a series of numerical simulations. It is necessary, therefore, to confirm such values by means of the analysis of existing compressors and turbines. Garrett data relative to several turbo machinery of different size have been used for this validation step. The comparison was carried out on the basis of the original operative maps, courtesy of Garrett (Figures 3 and 4). Provided a machine of known geometry and with known operative maps is available (in our case, the Garrett GT12, C224/45 trim compressors and GT12, T229/72 trim turbine), the method adopted to collect and compare the available compressor data is the following:

(1) Based on the compressor/turbine map of the selected machine, calculate Re with Equation (2);

(2) With the design value for the rpm, calculate the specific speed to enter the map;

(3) Draw on the map the curve for that specific speed " $n$ s", and extrapolate for every value of the flow rate, the compression/expansion ratio and the efficiency;

(4) Calculate the corrected flow rate accordingly;

(5) Use Equation (3) to calculate the reference efficiency $\eta_{\text {ref; }}$

(6) Use $\eta_{\text {ref }}$ to calculate, with Equation (5), the polytropic efficiency $\eta_{\text {pol; }}$;

(7) Calculate the polytropic work;

(8) Finally, compute $n_{\mathrm{s}}$ and $\mathrm{d}_{\mathrm{s}}$.

GT1241, 72 Trim, 0.43 A/R

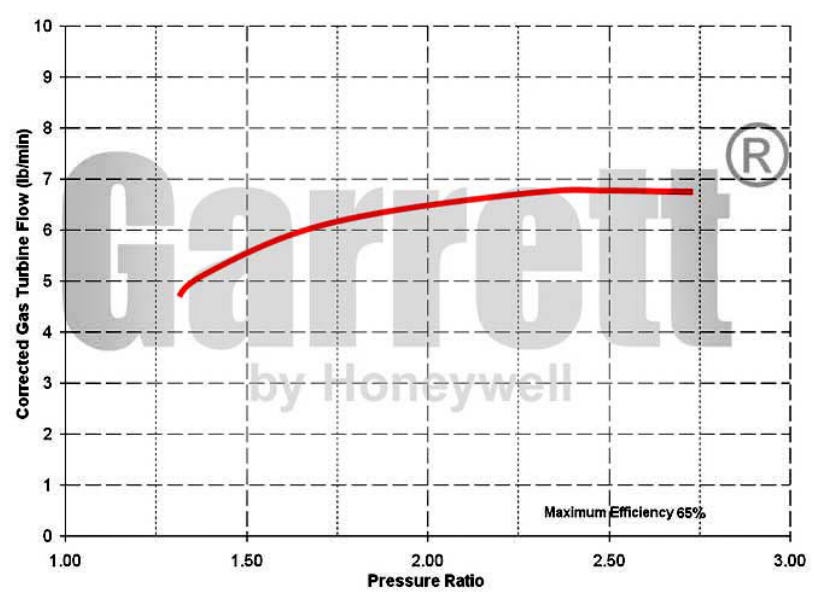

Figure 4. Typical Honeywell/Garrett ${ }^{\circledR}$ turbine maps. Reprinted with permission from [17]. 


\subsection{Compressor Maps}

Three different GT devices, all manufactured by the Garrett Corporation, have been considered in this work, identified by their impeller exit diameter, $D=32,34,38 \mathrm{~mm}$ respectively. The graphs are reported in Figure 5. Following the suggestions of one of the reviewers, the validation was extended by use of additional maps by Mitsubishi \& KKK. We compared some of the Mitsubishi maps (for the TD04-09B, -13G and -15G) and the KKK 04-14 (Figure 6) with the original Garrett maps and found that all of them cover a similar Re range and that the impellers sizes are very similar, which was to be expected (Figure 7).

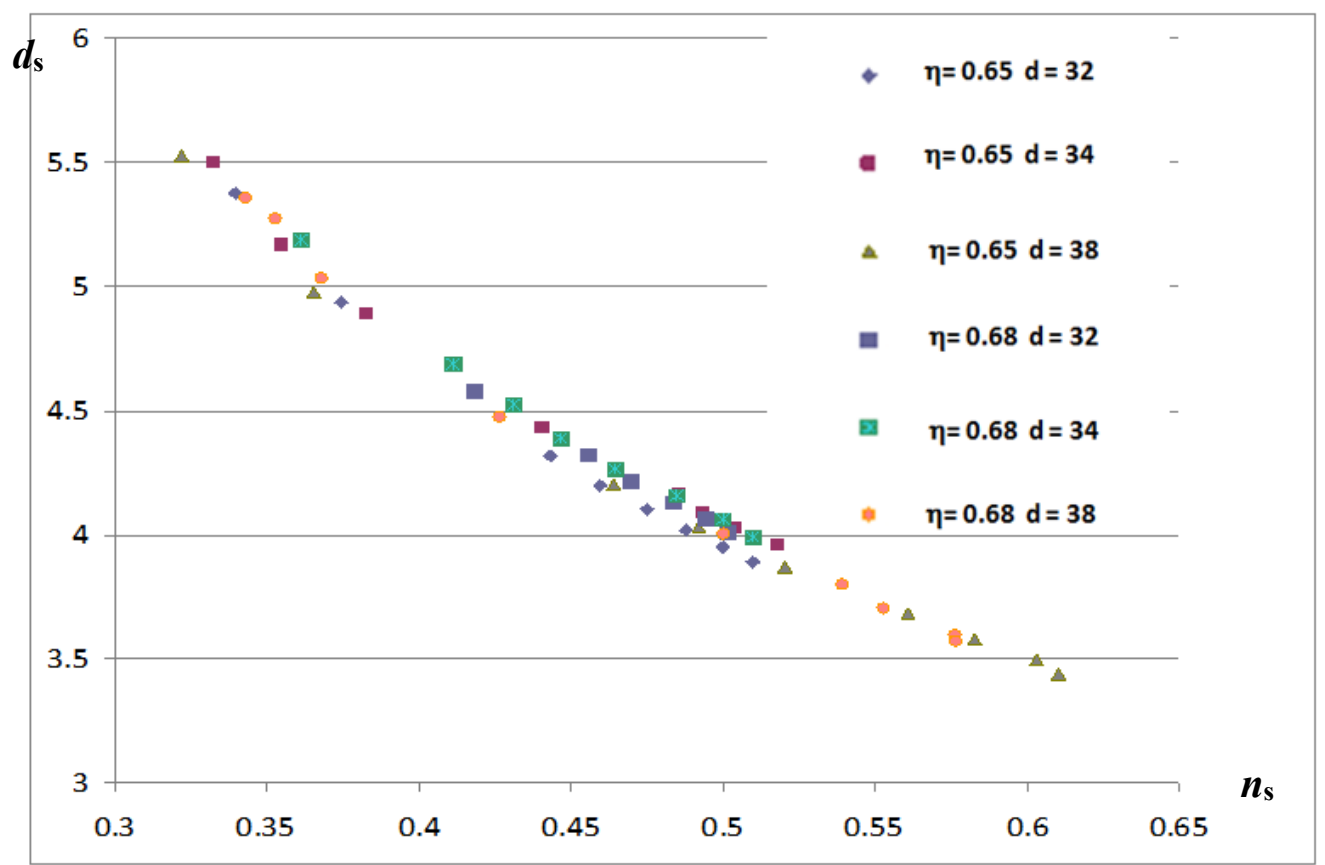

(a)

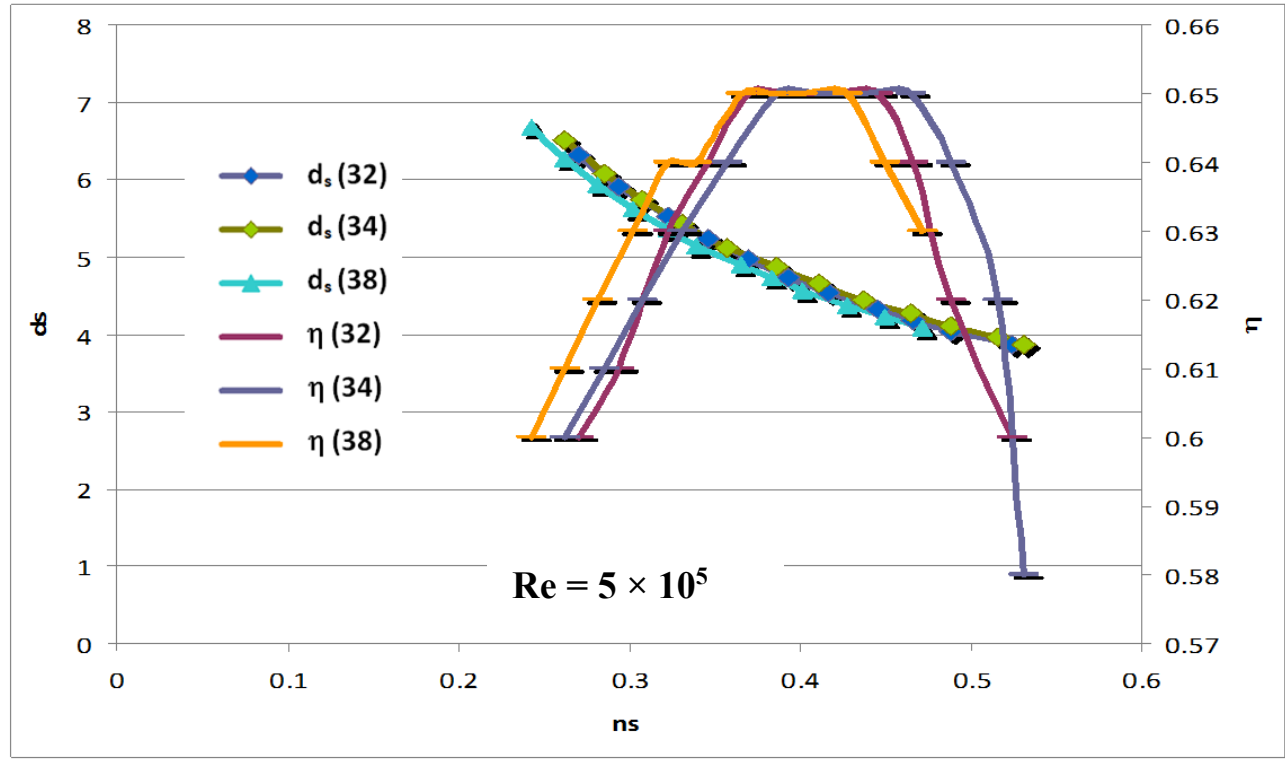

(b)

Figure 5. (a) Iso-efficiency points (Cordier line) for different compressor impeller diameter; and (b) $d_{\text {s }}$ and efficiency curves for a fixed Re and different compressor impeller diameter. 


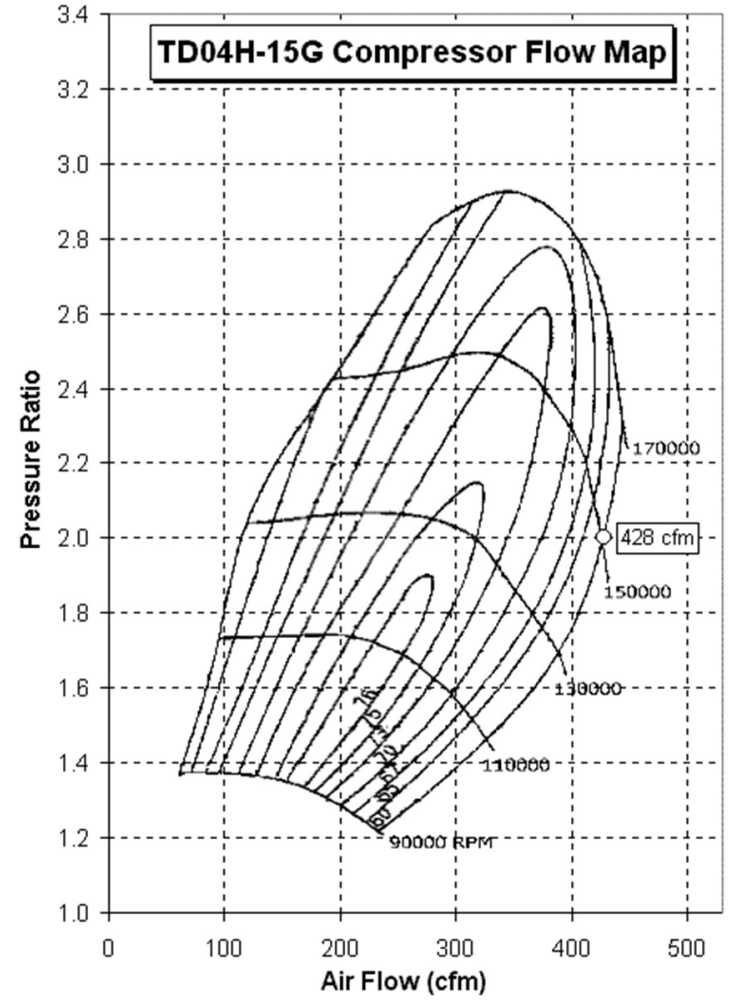

(a)

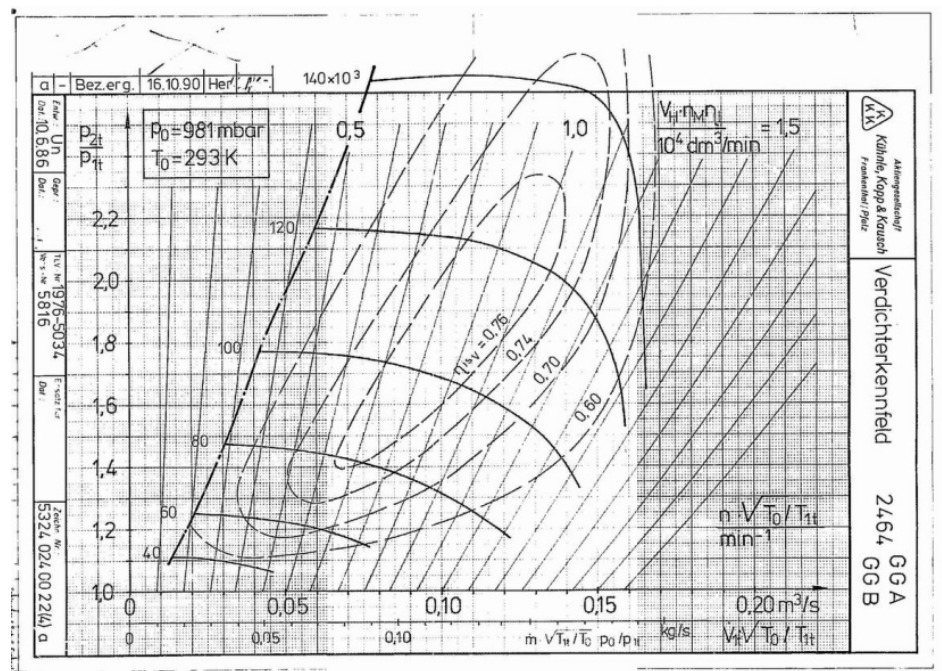

(b)

Figure 6. Compressor maps for (a) the Mitsubishi TD04 09B and (b) the KKK 04-14 used for the comparison and evaluation of proposed Equation (5).

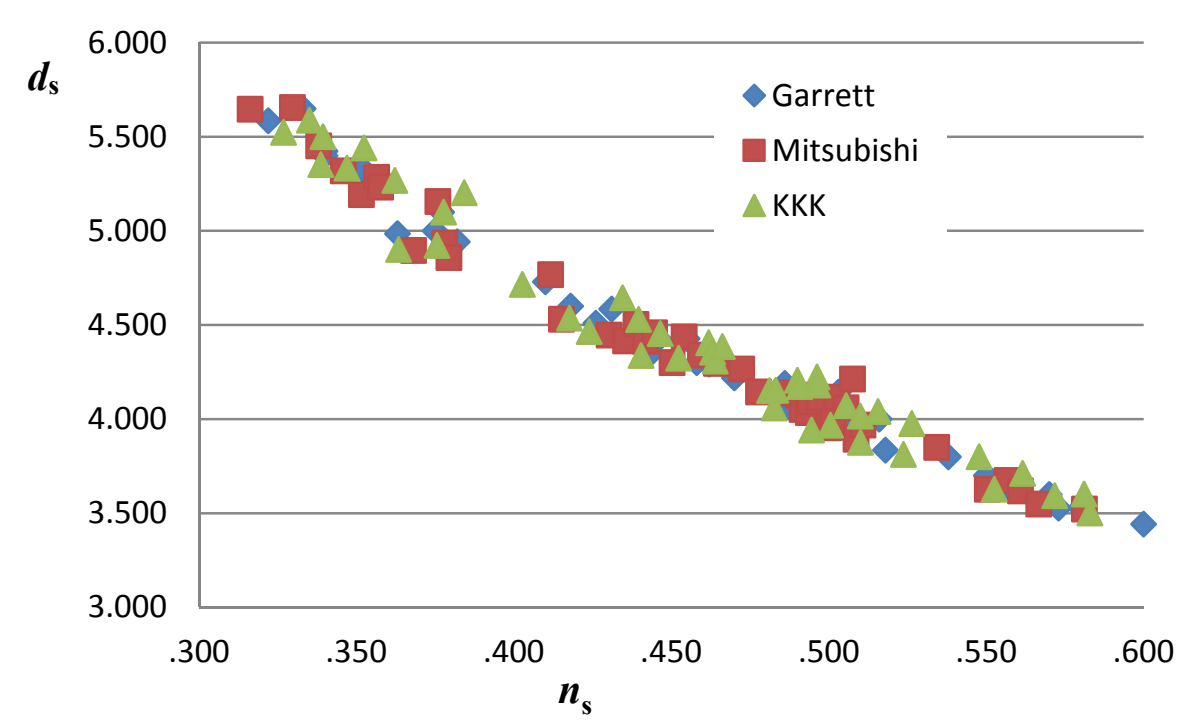

Figure 7. Comparison of the trends of the $\eta_{\max }$ of three different compressors.

The very good agreement between the predictions of Equation (5) and the available experimental data confirms the validity of the proposed efficiency scaling. Figure 5a shows the trend of the curves of iso-efficiency for three different diameters $(32,34$ and $38 \mathrm{~mm})$ of the compressor impeller, for several values of the Reynolds number. Here we only report two different values of the efficiency, corresponding to $\mathrm{Re}=5 \times 10^{5}$ and $7 \times 10^{5}$, and their dispersion for different values of ns at design rotational speed $(\omega=15,708 \mathrm{rad} / \mathrm{s}$, or $150,000 \mathrm{rpm})$. Figure $5 \mathrm{~b}$, relative to a single Re value (in the 
validation process, these considerations have been repeated for every value of Re), shows the efficiency curves corrected according to Equation (5) and the $d_{\mathrm{s}}$ curves as functions of $n_{\mathrm{s}}$. Figure 8 illustrates "the corrected" optimal curves in the $n_{\mathrm{s}} / d_{\mathrm{s}}$ plane for three different values of Re: these curves were calculated with Equation (5). The same procedure was repeated for the remaining compressor maps. Figure 9 shows the values obtained using the Mitsubishi and KKK compressor maps respectively. Different diameters have been considered, as well as different Reynolds numbers, while the design angular velocity remains constant at $\omega=15,708 \mathrm{rad} / \mathrm{s}$. The results obtained using the proposed formula show a good correspondence with the experimental values: in our judgement, this is a direct consequence of the philosophy of our approach, which applies the scaling only to the Re depending portion of the correction factor. The factor $a$ does not change in Equation (3), so that the "inviscid" losses, which do not scale with Re, remain unchanged.

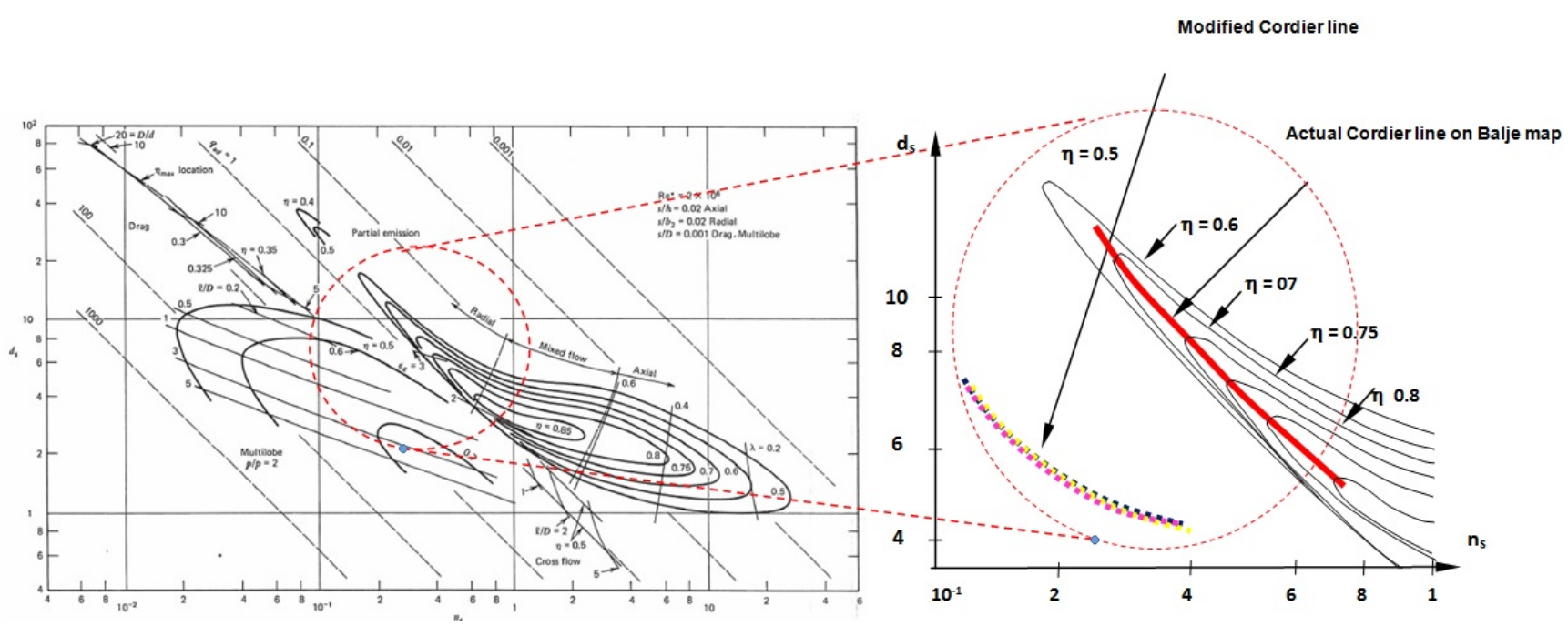

Figure 8. The low-Re Cordier curve on the standard Balje compressor map. Comparison between the actual Cordier line and the Modified Cordier line.

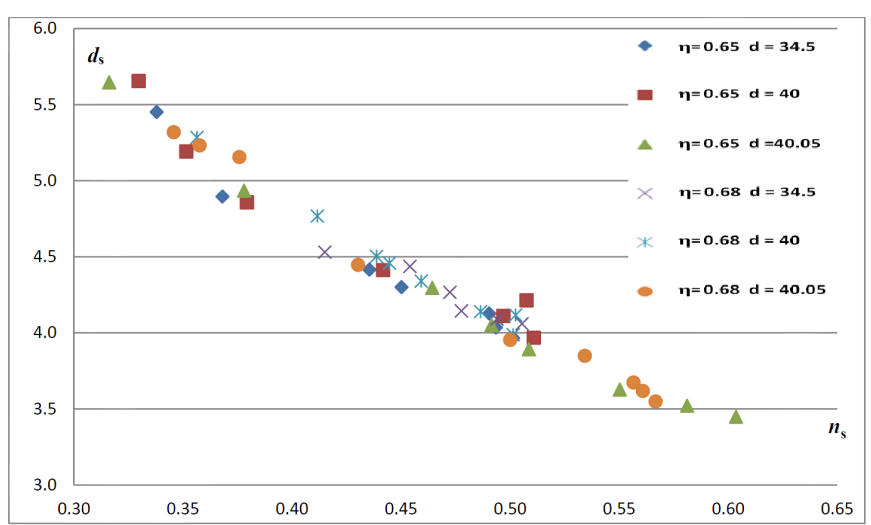

(a)

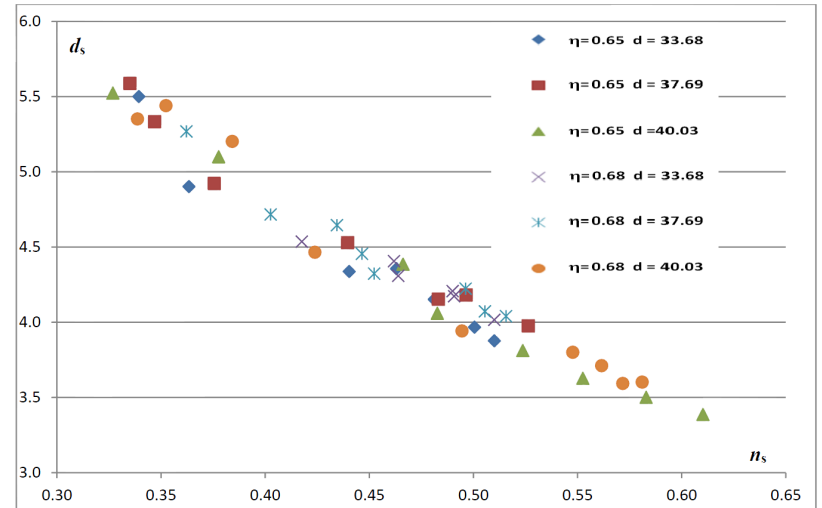

(b)

Figure 9. Iso-efficiency points (Cordier line) for different compressor impeller diameter: (a) Mitsubishi; and (b) KKK. 


\subsection{Turbine Maps}

Repeating the same procedure for radial turbines, we again obtained a good agreement between the predictions of Equation (5) and the experimental data referring to several commercial turbines. As for the compressors, the calculated $\eta=\mathrm{f}\left(n_{\mathrm{s}} / d_{\mathrm{s}}\right)$ curves lie in an area below the optimum and shifted to the left with respect to the peak values. These results are shown in Figure 10 only for Garrett turbines: they prove that - for a given turbine mass flow rate and turbine inlet temperature - either lower $\omega$ or higher specific work (i.e., a different blade design) must be adopted to attain the "optimal" efficiency, which is anyhow significantly lower than the one predicted by the Balje chart. Similar results and conclusions have been obtained for the Mitsubishi and KKK turbines.

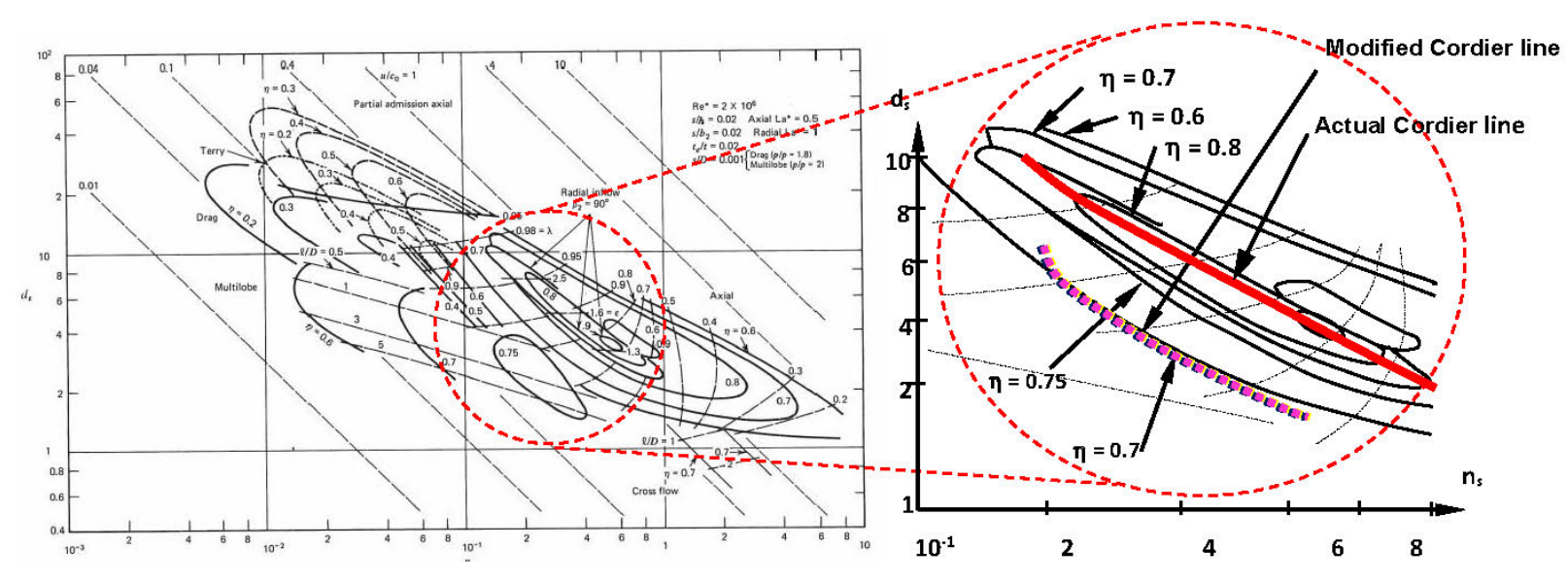

Figure 10. Radial turbines: the low-Re Cordier curve on the standard Balje turbine map. Comparison between the actual Cordier line and the Modified Cordier line.

\section{The Proposed Operational Approach for the Scaling-Down of the Balje Maps}

As previously stated, we assumed as a first approximation that the manufacturing process and clearances are compatible with the parameters in Balje charts: this is a necessary step to ensure congruent results. Next, we neglect relative roughness effects: this is not strictly necessary within the present framework but it is essential for simplifying the treatment discussed here so that attention is placed solely on the influence of the Reynolds number (as derived from numerical calculations). Since the newly derived $\eta=f(R e)$ correlation has been shown: (a) to be in reasonable accord with the numerical and experimental results; and (b) to lead to the Balje limit for $\mathrm{Re}=$ ReBalje, the following procedure can be proposed to extend into the ultra-micro scale the preliminary design selection at larger scales:

(1) On the basis of the actual design data, identify an optimal value of $n_{\mathrm{s}} / d_{\mathrm{s}}$ for radial compressors/turbines on the Balje chart;

(2) Calculate the Re of the micro-scale machine;

(3) Introduce an efficiency factor $<1$ given by Equation (5) that multiplies the total-to-static efficiency extracted from the chart;

(4) Calculate the Euler work from the design data and extract the angular velocity $\omega$ and the impeller diameter $D$ from $n_{\mathrm{s}}$ and $d_{\mathrm{s}}$; 
(5) Check that $U<U_{\max }$ (the allowable material stress limit for ultra-micro components may be higher): if this constraint is not abided by, choose a different $n_{\mathrm{s}} / d_{\mathrm{s}}$ pair and go back to Step (1);

(6) If the computed Re is different from the value assumed in Step (3), go back to Step (3), substitute the old Re number with the new one, and iterate.

\section{Conclusions}

The paper suggests a simple method to scale the Balje maps for low Re values. The method represents a generalization and a systematization of preliminary studies previously published on this topic by the authors' group. It is valid for adiabatic transformations, and thus its results can be directly compared to the classical Balje charts. Though the efficiency correction as a function of $\operatorname{Re}$ is completely similar to - and congruent with - the classical Stodola and Wiesner method, its novelty resides in a more accurate calculation of the correlation coefficients, which are obtained by a combination of numerical and laboratory experiments.

In spite of the relatively small number of CFD simulations, the results display a remarkably good agreement with the real operational map data of the devices object of the present study. This reinforces the belief in the correctness of the proposed preliminary design procedure for application to the small Re, ultra-micro scale range.

In particular, the results agree well with the available operational maps of a broad series of commercially available compressors and turbines. The most important practical result is the possibility of making a direct use of the Balje maps in the small-scale range by considering a Cordier line shifted towards lower $n_{\mathrm{s}}$ and $d_{\mathrm{s}}$ (or, for the same $n_{\mathrm{s}}$ and $d_{\mathrm{s}}$, towards lower efficiencies). The Re-dependence of the results is apparent, and Equation (5) constitutes a good and reliable starting point for a preliminary design procedure, which in followed in any case in real industrial cases by prototype testing.

\section{Acknowledgments}

The research has been partially sponsored by the Italian Army HQ within the framework of PNR 714.

\section{Author Contributions}

Roberto Capata's contribution dealt with the experimental tests and comparisons, while Enrico Sciubba was in charge of all aspects related to turbomachinery and CFD simulations.

\section{Conflicts of Interest}

The authors declare no conflict of interest.

\section{Nomenclature}

$\begin{array}{ll}\text { a } & \text { Constant in Equation (4) } \\ b & \text { Impeller width (m) } \\ \mathrm{c}, \mathrm{c}^{\prime} & \text { Constant in Equation (4) } \\ D & \text { Impeller diameter (m) } \\ d_{\mathrm{s}} & \text { Specific diameter }\end{array}$




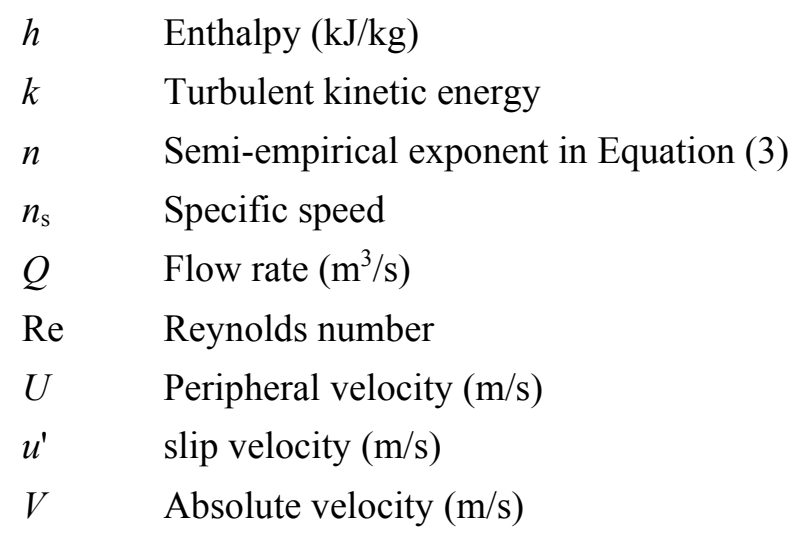

\section{Greek Symbols}

$\begin{array}{ll}\eta & \text { Efficiency } \\ v & \text { Kinematic viscosity }\left[\mathrm{m} / \mathrm{s}^{2}\right] \\ \rho & \text { Density }\left(\mathrm{kg} / \mathrm{m}^{3}\right) \\ \omega & \text { Angular velocity }[\mathrm{rad} / \mathrm{s}]\end{array}$

\section{Subscripts}

$\begin{array}{ll}1 & \text { Inlet } \\ 2 & \text { Outlet } \\ \mathrm{e} & \text { External } \\ \text { hyd } & \text { hydraulic } \\ \mathrm{p} & \text { Polytropic } \\ \text { ref } & \text { Reference }\end{array}$

\section{References}

1. Gong, X.; Chen, R. Total pressure loss mechanism of centrifugal compressors. Mech. Eng. Res. 2014, 4, 45-59.

2. Wiesner, F.J. A new appraisal of Reynolds number effects on centrifugal compressor performance. J. Eng. Gas Turbines Power 1979, 101, 384-396.

3. Stodola, A.; Lowestein, C. Steam and Gas Turbines; McGraw-Hill: New York, NY, USA, 1927; Volume 1.

4. Balje, O. Turbomachines; John Wiley \& Sons: New York, NY, USA, 1981.

5. Dixon, S.L. Fluid Mechanics and Thermodynamics of Turbomachinery, 3rd ed.; Pergamon Press: Exeter, UK, 1992; pp. 1-263.

6. Japikse, D. Centrifugal Compressor Design and Performance; Concept ETI Inc.: White River Junction, VT, USA, 1996.

7. Japikse, D.; Baines, N.C. Introduction to Turbomachinery; Concept ETI Inc.: White River Junction, VT, USA, 1997.

8. Casey, M.V. The effects of Reynolds number on the efficiency of centrifugal compressor stages. J. Eng. Gas Turbines Power 1985, 107, 541-548. 
9. Strub, R.A.; Bonciani, L.; Borer, C.J.; Casey, M.V.; Cole, S.L.; Cook, B.B.; Kotzur, J.; Simon, H.; Strite, M.A. Influence of the Reynolds number on the performance of centrifugal compressor. J. Turbomach. 1987, 109, 541-544.

10. Peirs, J.; Reynaerts, D.; Verplaetsen, F. A Microturbine for Electric Power Generation. J. Sensors Actuators A Phys. 2004, 113, 86-93.

11. Capata, R.; Sciubba, E.; Silva Caaveiro, G. Effects of the Reynolds Number on the Efficiency of an Ultra-micro Compressor. In Proceedings of the ECOS 2007, Padova, Italy, 25-28 June 2007.

12. Ishihama, M.; Sakai, Y.; Matsuzuki, K.; Hikone, T. Structural Analysis of Rotating Parts of an Ultra Micro Gas Turbine. In Proceedings of the International Gas Turbine Congress, Tokyo, Japan, 2-7 November 2003.

13. Capata, R.; Sciubba, E. Use of Modified Balje Maps in the Design of Low Reynolds Number Turbo Compressors. In Proceedings of the ASME 2012 International Mechanical Engineering Congress and Exposition-IMECE Conference, Houston, TX, USA, 9-15 November 2012; pp. 835-841.

14. Silva Caaveiro, G. Micro Gas Turbines Numerical Analysis. Master's Thesis, University of Roma "Sapienza", Roma, Italy, June 2007.

15. Epstein, A.H. Millimetre-Scale, MEMS Gas Turbine Engines. In Proceedings of the ASME Turbo Expo 2003, Atlanta, GA, USA, 16-19 June 2003.

16. Iandoli, C.L.; Sciubba, E. 3-D numerical calculation of the local entropy generation rates in a radial compressor stage. Int. J. Thermodyn. 2005, 8, 83-94.

17. Turbocharger Specs. Garrett GT20-GT2052-52 TRIM-225HP. Available online: http://turbochargerspecs.blogspot.it/2011/02/garrett-gt20-gt2052-225-hp.html (accessed on 15 May 2015).

(C) 2015 by the authors; licensee MDPI, Basel, Switzerland. This article is an open access article distributed under the terms and conditions of the Creative Commons Attribution license (http://creativecommons.org/licenses/by/4.0/). 